\title{
Characterization of Gelatin/CMC Scaffolds by Electrospinning and Comparison with Freeze Dry Techniques
}

\author{
Kaona Jongwuttanaruk, Prayoon Surin, and Fasai Wiwatwongwana
}

\begin{abstract}
Gelatin/CMC mixture was used to produce nanofiber scaffold in order to analyze fiber's physical characteristic. The gelatin solution made from dissolving gelatin in water which serves as solvent for the solution because water is a great solvent for gelatin. However, the solution used in fiber production with electrospinning, this research used organic solvent, 2,2,2-trifluoroethanol, which is also a good solvent for gelatin and hence produces good raw material for fiber production using electrospinning. However, since CMC was unable to dissolved under organic solvent like 2,2,2-trifluoroethanol, it was dissolved in water instead. The weight ratios of the gelatin/CMC mixture are 100/0, 90/10, 80/20, $70 / 30$ and $60 / 40$, respectively. Whereas higher or lower concentration of the solution resulted in failure to produce the fiber by using electrospinning. After nanofiber was produced, the size of the fiber was between $36-563 \mathrm{~nm}$ and this research also found that the scaffold of the $60 / 40$ mixture occurred the smallest fiber with average fiber size of $41 \mathrm{~nm}$ which contained white granules in its structure. Water absorption analysis of fiber revealed that $100 / 0$ mixture scaffold had the highest rate of absorption which was $11.79 \%$ and average lower swelling ratio of $62.7 \%$ in comparison with swelling ratio of the scaffold from freeze dry technique.
\end{abstract}

Index Terms - Scaffold, Gelatin, carboxymethylcellouse, electrospinning, freeze dry.

\section{INTRODUCTION}

Electrospinning is a fiber production method that can produce extremely small, in the order of nanometer, fiber from synthetic polymer or natural polymer. Electrospinning uses the principle of difference in potential [1] to deliver polymer to the collector. The size of the fiber is depended on the voltage, material's flow rate, distance between the injector and the collector for fiber and concentration of the material. The result of electrospun fiber can be in the form of non-woven fiber and aligned fiber. Electrospinning is popular among various industries, including in medicine such as for production of prosthetic skin and wound dressing material [2].

Manuscript received January 30, 2018; revised March 28, 2019. This work was supported in part by Department of Advanced Manufacturing Technology, Faculty of Engineering, Pathumwan Institute of Technology, Thailand

The authors are with Department of Advanced Manufacturing Technology, Faculty of Engineering, Pathumwan Institute of Technology, 833 Rama 1 Road, Wangmai, Pathumwan, Bangkok 10330, Thailand (e-mail: Kaonadod@gmail.com, Prayoon99@gmail.com, fasaiw227@gmail.com).
Mostly, production of fiber by electrospinning will use degradable polymer, and will focus on synthetic polymer such as PLA, PGA, PLGA and PCL as well as using natural polymer for medical application. This is because natural polymer can be easily accepted into human's body [3]. Gelatin is a type of natural biopolymer and can be extracted from collagen which is a substance that can be found abundantly in animal's skin, meat and bone. Gelatin also has another special characteristic such as highly biodegradable material and even though gelatin is hydrophilic polymer. It cannot be mixed and formed into fiber by electrospinning. Therefore, this research used a solvent that could efficiently dissolve gelatin, 2,2,2 trifluoroethanol with the weight/volume percentage concentration of $10 \mathrm{wt} \%$ [4].

However, because gelatin is quite expensive; in order to reduce the cost of prosthetic skin, it is necessary to mix gelatin with other cheaper natural polymer. Therefore, carboxymethyl cellulose or CMC is an interesting candidate in this research. It can improves the strength of prosthetic skin as well. This is because CMC is being used widely in many industries such as in industrial production of detergent, paint, glue, fabric, paper, ceramic, food and medicine. Moreover, CMC is colorless, odorless, does not present any harmful effect to human and a very efficient water soluble substance [5]. CMC or cellulose derivative is generally made of cellulose tissue that has high amount of alpha cellulose which is generally known as high quality cellulose and it is also cheaper than gelatin.

Moreover, in order to increase the fiber's mechanical strength, this research applied the crosslinking technique by using high temperature or dehydrothermal treatment (DHT). DHT is a physical process which concerns giving high thermal energy under vacuum to the material. Normally, this process used over $100^{\circ} \mathrm{C}$ which extracted water from the fiber and cause condensation process that could produce the crosslinking of material and strengthens the fiber [6].

\section{EXPERIMENTAL DETAILS}

\section{A. Materials}

Gelatin from Porcine Skin, 180 G Bloom, in powder form, Type B, from Fluka Analytical and 2,2,2-trifluoroethanol (TFE) (purity 99.0\%) from Sigma-Aldrich were prepared. Preparation of gelatin solution was made by dissolving gelatin in TFE and stirred the solution at room temperature for 6 hours at the concentration of $10 \%$. Then powdered CMC 
from Sigma-Aldrich was dissolved in distilled water, at the concentration of $0.8 \%$ and stirred at room temperature for 30 minutes. Higher or lower concentration of the solution resulted in failure to produce the fiber by using electrospinning. Afterward, the solution were prepared for fiber production using electrospinning where gelatin solution and CMC solution were mixed at the ratio of 100/0, 90/10, $80 / 20,70 / 30$ and $60 / 40$ and then stirred at room temperature for 30 minutes.

\section{B. Electrospinning}

Gelatin/CMC solutions were prepared at the ratios of 100/0, 90/10, 80/20, 70/30 and 60/40 for fiber production by using electrospinning process was schematically shown in Fig. 1. The mixture was added into $5 \mathrm{ml}$ syringe and the syringe was then attached to needle spinneret \#20 with the hole size of 0.9 $\mathrm{mm}$. The flow was controlled using syringe pump (KD-100, KD Scientific, Inc., USA) at the rate of $0.8 \mathrm{ml} / \mathrm{h}$. High voltage electricity was supplied from high voltage power supply (RR501.25R/230/DDPM, Gamma High Voltage Research, USA) at $1 \mathrm{kV}$. The mixture was then injected onto collector which was made of foil sheet. The distance between the needle's end to the foil sheet was 13 centimeters.

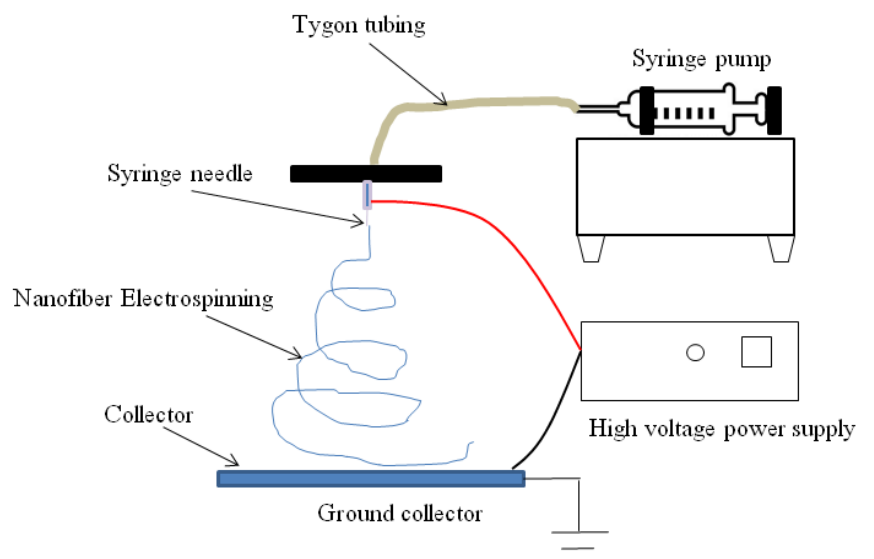

Fig. 1. Schematic diagram of electrospinning apparatus.

\section{Dehydrothermal Treatment (DHT)}

The resulted scaffold was then put into crosslinking technique by using DHT. The scaffold of gelatin and CMC mixtures at the ratio of 100/0, 90/10, 80/20, 70/30 and 60/40 was put into desiccator with silica gel in vacuum container for 2 days. Then the scaffold was put into vacuum oven under the temperature of $140{ }^{\circ} \mathrm{C}$ for 48 hours.

\section{Characterization of Nanofibrous Scaffolds}

\section{1) Surface morphology of nanofibrous scaffolds}

The scaffold was analyzed its morphology by using scanning electron microscopy (SEM, JSM-5610LV, JEOL) at the voltage of $20 \mathrm{kV}$ after it has been coated with gold [7]. The fiber's thicknesses were also measured for at least 20 values and then calculated for the average thickness.

The morphology of the scaffold fabricated from electrospinning technique was shown in Fig. 2. When CMC was mixed into gelatin at different ratios, we found that the size of fiber and structure of scaffold was changed as well.
The more CMC was added into the mixture, the smaller the fiber would be and CMC would be embedded into the fiber. In Fig. 2E which depicted the result of gelatin and CMC mixture at the ratio of 60/40, the fiber was quite small and contained several white granules all over the scaffold. Whereas the structure of scaffolds fabricated by freeze dry technique had porous structure. It was found that gelatin and CMC mixture at the ratio of $100 / 0$, the porous of scaffold was quite small and the ratio of $80 / 20$ showed the biggest pore [8].

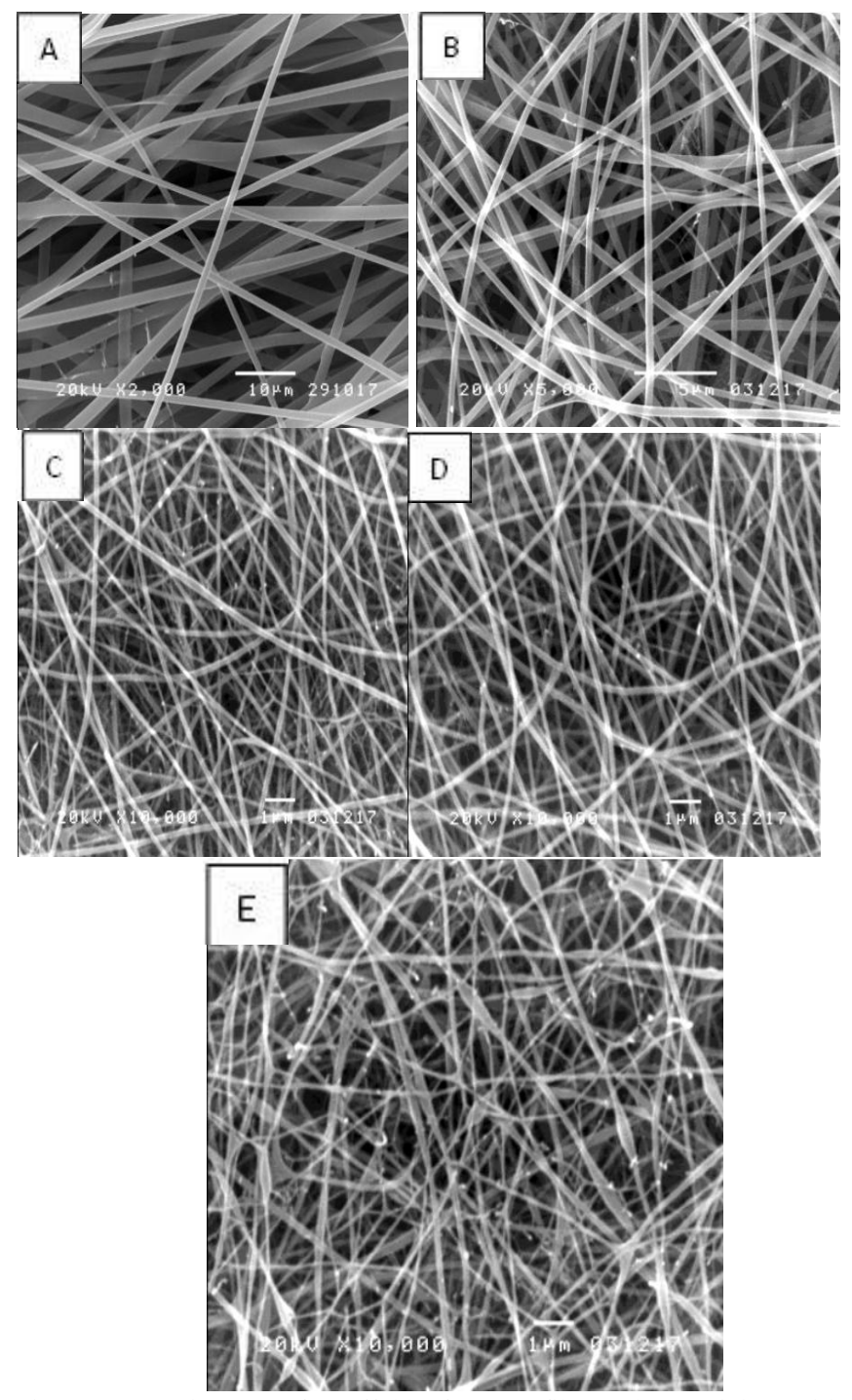

Fig. 2. SEM images of scaffolds for nanofibers electrospining with Gelatin/CMC ratios of 10/0 (A), Gelatin/CMC ratios of 90/10 (B), Gelatin/CMC ratios of 80/20 (C), Gelatin/CMC ratios of 70/30 (D), Gelatin/CMC ratios of 90/10 (E) and all condition were DHT for 48h.

Table I revealed that when CMC increased from 0 to 40 percent, fiber's size was decreased from $597 \mathrm{~nm}$ to $41 \mathrm{~nm}$.

TABLE I: FiBER DiAMETERS OF ELECTROSPUN GELATIN/ CMC

\begin{tabular}{lllll} 
Gelatin/CMC & $\begin{array}{l}\text { Range of diameter } \\
(\mathrm{nm})\end{array}$ & $\begin{array}{l}\text { Average } \\
(\mathrm{nm})\end{array}$ & diameter \\
\hline
\end{tabular}

\begin{tabular}{lcc}
\hline $10 / 0$ & $563-627$ & 597 \\
\hline $90 / 10$ & $307-444$ & 378 \\
\hline $80 / 20$ & $113-140$ & 125 \\
\hline $70 / 30$ & $77-92$ & 80 \\
\hline $60 / 40$ & $36-46$ & 41 \\
\hline
\end{tabular}




\section{2) Swelling test}

The scaffold was put into the swelling test in order to find the difference of dry weight and wet weight of the scaffold in percentage. The dried scaffold was weighted and then soaked the scaffold under PBS buffer solution at the $\mathrm{pH}$ of 7.4 and temperature of $37{ }^{\circ} \mathrm{C}$ for 3 hours [9]. Then both sides of the scaffold were wiped with low lint paper 10 seconds for each side and then weighted immediately. The dry weight and wet weight were then calculated into percentage using the following formula as shown in (1). We then compared the resulted of swelling ratio of scaffolds from electrospinning and freeze dry technique [10].

$$
\text { Swelling ratio }=\frac{W_{s o}-W_{0}}{W_{0}}
$$

where as

$W_{s o}$ is the weight of scaffold after its water content was absorbed

$W_{0}$ is the initial weight of the scaffold

Fig. 3 revealed that the scaffolds from electrospinning with the mixture of 100/0 ratio had swelling ratio of $11.79 \%$ which was the highest. All scaffolds from electrospinning had average lower in swelling ratio compared with the scaffolds from freeze dry technique, except for the case of gelatin and CMC mixture at the ratio of 90/10 which was higher than freeze dry technique. Meanwhile, the highest swelling ratio from freeze dry technique was from gelatin and CMC mixture at the ratio of 80/20 which was $44.67 \%$ [11].

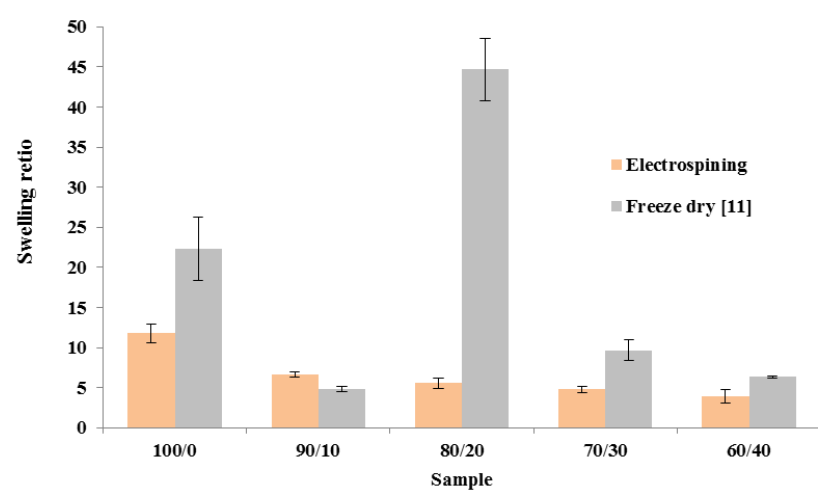

Fig. 3. Comparison of swelling ratio of Gelatin/CMC scaffold by Electrospinning and freeze dry.

\section{CONCLUSION}

Gelatin/CMC fiber produced by electrospinning technique revealed that the highest in swelling ratio and the largest of fiber size was found in gelatin and CMC mixture at the ratio of $100 / 0$. Adding CMC into the gelatin fiber occurred in smaller in fiber size and lower in swelling ratio compared with pure gelatin fibrous scaffold. We could summarize that size of the fiber effected to the swelling ratio which the smaller of fiber, the lower of swelling ratio was found.

Analyzing the nanofibrous scaffold produced by using electrospinning technique, the gelatin/CMC fiber with large fiber size would also has high swelling ratio. In similar to the scaffold from freeze dry technique, the scaffold with the mixture of gelatin and CMC at the ratio of $80 / 20$ had the largest pore in the structure and also showed the highest swelling ratio which was $44.67 \%$. Comparison of fiber production using electrospinning and porous scaffold from freeze dry technique, the average swelling ratio of fiber from electrospinning was $62.7 \%$ which lower than the scaffold from freeze dry technique.

\section{ACKNOWLEDGMENT}

This research conducted under the support of Department of Advanced Manufacturing Technology and Department of Manufacturing Engineering, Faculty of Engineering, Pathumwan Institute of Technology was gratefully acknowledged for provided facilities and experiment being required for this research.

\section{REFERENCES}

[1] Z.-M. Huanga, Y. Z. Zhang, S. Ramakrishna, and C. T. Lim, "Electrospinning and mechanical characterization of gelatin nanofibers," Polymer, vol. 45, pp. 5361-5368, 2004.

[2] Y. Z. Zhang, J. Venugopal, Z.-M. Huang, C. T. Lim, and S. Ramakrishna, "Crosslinking of the electrospun gelatin nanofibers," Polymer, vol. 47, pp. 2911-2917, 2006.

[3] E. J. Chong, T. T. Phan, I. J. Lim, Y. Z. Zhang, B. H. Bay, S. Ramakrishna, and C. T. Lim, "Evaluation of electrospun PCL/gelatin nanofibrous scaffold for wound healing and layered dermal reconstitution," Acta Biomaterialia, pp. 321-330, 2007.

[4] M. Pezeshki-Modaress, H. Mirzadeh, and M. Zandi, "Gelatin-GAG electrospun nanofibrous scaffold for skin tissue engineering: Fabrication and modeling of process parameters," Materials Science and Engineering $C$, vol. 48, pp. 704-712, 2015.

[5] F. Wiwatwongwana and N. Promma, "Evaluation of Gelatin / Carboxymethylcellulose scaffolds using mooney-rivlin model," KMITL Sci. Tech. J., vol. 15, no. 2, 2015.

[6] S. R. Gomes, G. Rodrigues, G. G. Martins, C. M. R. Henriques, J. C. Silva, "In vitro evaluation of crosslinked electrospun fish gelatin scaffolds," Materials Science and Engineering C, vol. 33, pp. 1219-1227, 2013.

[7] H. Chen, J. Huang, J. Yu, S. Liu, P. Gu, "Electrospun chitosan-graft-poly ( $\Sigma$-caprolactone) /poly ( $\Sigma$-caprolactone) cationic nanofibrous mats as potential scaffolds for skin tissue engineering," International Journal of Biological Macromolecules, vol. 48, pp. 13-19, 2011.

[8] F. Wiwatwongwana and S. Pattana, 2011, "Modification gelatin scaffold with carboxymethylcellulose for dermal skin," in Proc. the 5th International Conference on Bioinformatics and Biomedical Engineering, 10-12 May, 2011, Wuhan, China, pp. 1-4.

[9] J. S. Mao, L. G. Zhao, Y. J. Yin, K. D. Yao, 2003, "Structure and properties of bilayer chitosan-gelatin scaffolds," Biomaterials, vol. 24, pp. 1067-1074.

[10] M. Okhawilai, R. Rangkupan, S. Kanokpanont, and S. Damrongsakkul, "Preparation of Thai silk fibroin/gelatin electrospun fiber mats for controlled release applications," International Journal of Biological Macromolecules, vol. 46, pp. 544-550, 2010.

[11] F. Wiwatwongwana, and S. Pattana, 2011, "Influence of Blending Carboxymethylcellulose with Gelatin Scaffold on Mechanical Properties," in Proc. The 2nd TSME International Conference on Mechanical Engineering, Sheraton Krabi Beach Resort, Krabi, Thailand, October 19-21, 2011.

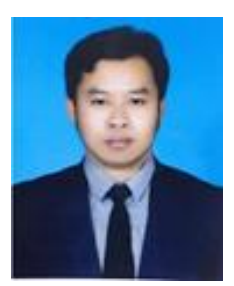

Kaona Jongwuttanaruk was born in Sept. 12, 1980 in Thailand. He is a Ph.D candidate at Pathumwan Institute of Technology, Thailand.

He graduated with a master of engineer in industrial engineering in April 2014 from Rajamangala University of Technology Thanyaburi, Patumtani, Thailand. And he got the bachelor of engineer in industrial engineering in April 2004 from Eastern Asia University, Patumtani, Thailand.

$\mathrm{He}$ has experience in engineering manager at Department, Unity Electronics Co., Ltd. He was an assistance engineering manager at Chanon Assy Co., Ltd. He was also a Sr. process engineer at Yoneden Ltd, Thailand. 


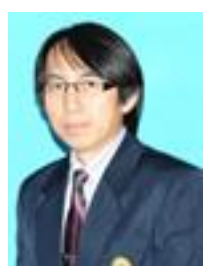

Prayoon Surin was born on April 16, 1972 in Thailand. He graduated with a Ph.D. of integrated product design and manufacturing in 2013 from King Mongkut's University of Technology Thonburi, Bangkok, Thailand, a master of materials technology in 2001 form King Mongkut's University of Technology Thonburi, Bangkok, Thailand, he also got a bachelor of industrial engineering in 2003 from Rajamangala University of Technology Thanyaburi, Pathumthani, Thailand.

He has teaching experiences as a lecturer at the Department of Industrial Engineering, Faculty of Engineering, Pathumwan Institute of Technology from 1998 to present.

He has published many papers such as : T. Thongkanluang, K. Buasri, P. Surin, N. Chirakanphaisarn, S. Jakthin, and P. Kahawong, "Physical and mechanical properties of fiber boards from oil palm empty fruit bunch fibers mixed with water hyacinth fibers," SNRU Journal of Science and Technology, vol. 10, no. 1, pp. 52-57, January-April, 2018; D. Buntam, W. Permpoonsinsup, and P. Surin, "Optimal mixture experiment design using neural network," IEET International Electrical Engineering Transactions, vol. 2, no. 2, p. 3, July-December, 2016.

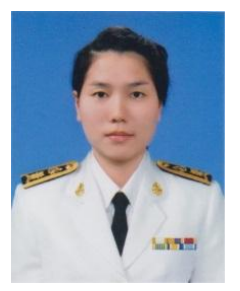

Fasai Wiwatwongwana was born on November 5, 1982 in Chiang Mai Province, Thailand. About her education, she graduated with a Ph.D. of mechanical engineering in December 2012 from Chiang Mai University, Chiang Mai, Thailand, master of chemical engineering in April 2007 from Chulalongkorn University, Bangkok, Thailand and bachelor of engineering, chemical engineering in April 2004 from Thammasat University, Bangkok, Thailand.

She has teaching experiences as a lecturer at the Department of Manufacturing Engineering, Faculty of Engineering, Pathumwan Institute of Technology from June 2014 to present, Department of Mechanical Engineering, Faculty of Engineering, Rajamangala University of Technology Lanna Chiang Mai from June 2013 to May 2014 and Biomedical Engineering Program (International Program), Graduate School,
Chiang Mai University from June 2009 to May 2013. She also has experiences as a researcher at Biomedical Engineering Center, Chiang Mai University from March 2008 to May 2013 and Center for Agricultural Biotechnology, Faculty of Agriculture, Chiang Mai University from July 2007 to February 2008.

Her publications are F. Wiwatwongwana, and N. Promma, "Evaluation of gelatin/carboxymethylcellulose scaffolds using mooney-rivlin model," KMITL Sci. Tech. J., vol. 15, no.2, pp. 70-79, Jul.-Dec. 2015 and F. Wiwatwongwana, Y. Khunathon, W. Rangsri, N. Promma, and S. Pattana, "Identification of shear modulus of gelatin blended with carboxymethylcellulose scaffolds using curve fitting method from compressive test," Journal of Materials Science Research, vol. 1, no. 4, pp. 106-113, 2012. 